\title{
Thrombotic microangiopathies during pregnancy : The obstetrical and neonatal perspective
}

\section{Sarno, Laura}

2019-06

Sarno , L , Stefanovic , V , Maruotti , G M , Zullo , F \& Martinelli , P 2019 , ' Thrombotic microangiopathies during pregnancy : The obstetrical and neonatal perspective ', European Journal of Obstetrics, and Gynecology ,and Reproductive Biology , vol. 237 , pp. 7-12 . https://doi.org/10.1016/j.ejog

http://hdl.handle.net/10138/318790

https://doi.org/10.1016/j.ejogrb.2019.03.018

publishedVersion

Downloaded from Helda, University of Helsinki institutional repository.

This is an electronic reprint of the original article.

This reprint may differ from the original in pagination and typographic detail.

Please cite the original version. 
Review article

\title{
Thrombotic microangiopathies during pregnancy: The obstetrical and neonatal perspective
}

\author{
Laura Sarno ${ }^{\mathrm{a}, *}$, Vedran Stefanovic ${ }^{\mathrm{b}}$, Giuseppe M. Maruotti ${ }^{\mathrm{a}}$, Fulvio Zullo ${ }^{\mathrm{a}}$, \\ Pasquale Martinelli ${ }^{\mathrm{a}}$ \\ a Department of Neurosciences, Reproductive and Dentistry Sciences, University Federico II, Via Pansini 5, 80131, Naples, Italy \\ ${ }^{\mathbf{b}}$ Department of Obstetrics and Gynecology, Fetomaternal Medical Center, Helsinki University Hospital and University of Helsinki, Haartmaninkatu 2, 00290, \\ Helsinki, Finland
}

\section{A R T I C L E I N F O}

\section{Article history:}

Received 24 February 2019

Received in revised form 18 March 2019

Accepted 18 March 2019

Keywords:

Thrombotic microangiopathies

Pregnancy

Maternal and neonatal outcome

\begin{abstract}
A B S T R A C T
Thrombotic Microangiopathies during pregnancy and puerperium are very rare and, if undiagnosed, can be lifethreating. Pregnancy and postpartum can represent a trigger in predisposed patients. Therefore, obstetricians are usually the first to observe clinical symptoms and laboratory abnormalities suggestive of Thrombotic Microangiopathies.

The aim of this review is to briefly describe the obstetrical and perinatal outcome of these entities and highlight the clues for a correct diagnosis of pregnancy-related Thrombotic Microangiopathies.
\end{abstract}

(C) 2019 Elsevier B.V. All rights reserved.

\section{Contents}

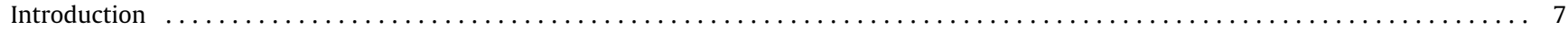

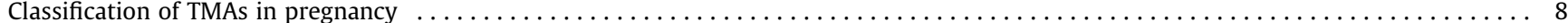

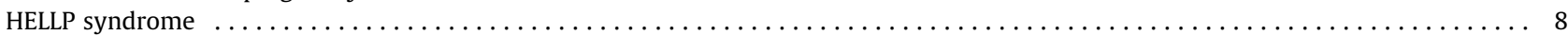

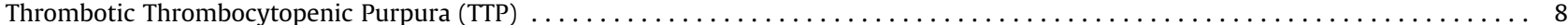

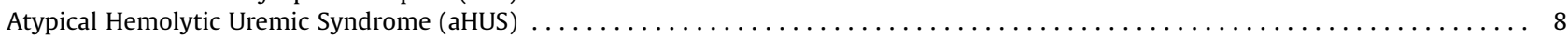

Differential diagnosis of thrombocytopenia in pregnancy and recognition of TMAs $\ldots \ldots \ldots$

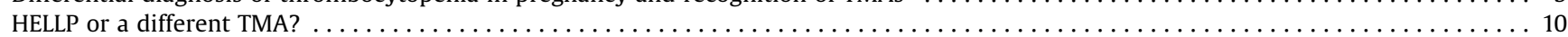

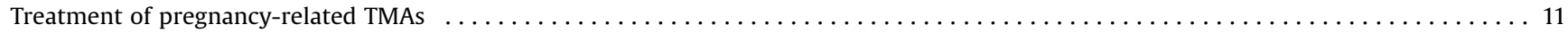

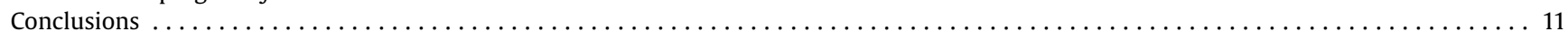

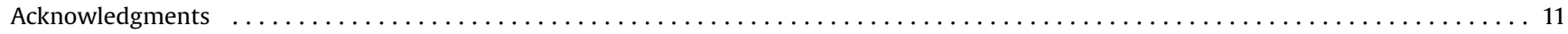

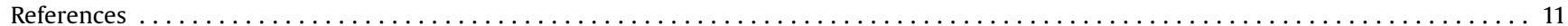

\section{Introduction}

Thrombotic microangiopathies (TMAs) are rare, severe conditions associated with serious morbidity and up to $90 \%$ mortality rate, if untreated. Prompt recognition and initiation of early specific therapy reduces substantially mortality rate to $10 \%-20 \%[1]$.

The term "thrombotic microangiopathy" defines a pathologic modification of the microvasculature, with detachment or swelling of the endothelium and luminal platelet aggregation leading to

\footnotetext{
* Corresponding author.

E-mail address: laura.sarno@unina.it (L. Sarno).
}

impairment of vessel microcirculation. The clinical consequences of these disorders are thrombocytopenia, mechanical haemolytic anemia and ischemic injuries to different organs, particularly affecting central nervous system and kidneys [2].

There is a wide range of diseases that can present with a microangiopathic phenotype; their laboratory features almost uniformly include thrombocytopenia and hemolytic anemia with evidence of red blood cell fragmentation, also identified as microangiopathic hemolytic anemia (MAHA) [2].

The two vulnerable periods that can act as trigger for development of TMAs are pregnancy and puerperium. Indeed, there is an immune adaptation during pregnancy, called materno-placental immune tolerance, characterized by an 
enhancement of the innate immune system and a suppression of the adaptive immune system. [3] The complement system has a central role in the innate immune system and its activation is controlled by several regulators in order to prevent an overactivation. An imbalance in its regulation can result in inflammation and thrombosis.

Pregnancy-related TMAs are rare conditions, with an incidence of $1: 25,000$ births [4]. However, they represent an obstetrical emergency and appropriate interventions are necessary to improve maternal and fetal outcomes. Therefore, differential diagnosis among pregnancy-related TMAs is crucial to ensure a correct management. In these cases, a cooperation of the multidisciplinary team (obstetrician, neonatologist hematologist, anesthesiologist, nephrologist and immunologist) is a cornerstone.

The aim of this review is not only to briefly describe obstetrical and perinatal outcomes of these entities, but also to highlight the clues for a correct diagnosis of pregnancy-related TMAs.

\section{Classification of TMAs in pregnancy}

There is an international consensus in defining under the name of TMAs three different conditions that can develop during pregnancy or puerperium: Haemolysis, Elevated Liver enzymes and Low Platelets (HELLP) syndrome, Thrombotic Thrombocytopenic Purpura (TTP) and atypical Hemolytic Uremic Syndrome (aHUS).

\section{HELLP syndrome}

The acronym HELLP defines a condition characterized by haemolysis $(\mathrm{H})$, elevated liver enzymes (EL), low platelet count (LP).

HELLP syndrome is the most common pregnancy specific TMA condition occurring in $0,2-0,6 \%$ of pregnancies [5]. A mortality rate ranging from 1 to $4 \%$ has been previously reported [6,7]; morbidity is related to complications, such as pulmonary edema, acute kidney injury (AKI), disseminated intravascular coagulation (DIC), placental abruption, liver hemorrhage, adult respiratory distress syndrome, stroke or sepsis [5].

Clinical symptoms include upper abdominal pain and tenderness, nausea, vomiting, headache and jaundice. Abdominal symptoms are more typical compared to other TMAs, but also neurological and renal damage can be present.

Although the pathogenesis of HELLP is believed to be on the spectrum of preeclampsia via abnormal placentation, endothelial dysfunction, and release of vasoactive substances in the first trimester, the cellular biology and natural history of the disease that results in its distinct phenotype is not well defined.

Recent evidence highlights complement activation in its pathogenesis. It has been postulated an up-regulation of the alternative pathway of complement using markers in serum and urine (C5b-9 or membrane attack complex/MAC) [8] and mutations in the activated protein C (APC) have been found in up to $20 \%$ of HELLP patients [9].

HELLP occurs typically during the third trimester, but it has reported to complicate pregnancy during the second trimester in up to $11 \%$ of cases. Pregnancy triggers HELLP in $69 \%$ of cases, while the rest of the cases appear postpartum, usually within $48 \mathrm{~h}$ after delivery [6].

Renal involvement and acute kidney injury (AKI) is observed in up to $40 \%$ of cases in otherwise healthy pregnant women [10], and progresses to end-stage renal disease (ESRD) in 10-30\% of cases [11].

Maternal mortality in AKI HELLP-related is reported to be $13 \%$ in a large prospective study conducted in the US on 442 pregnant women [6]. In the same study, $20 \%$ of postpartum HELLP cases were complicated by AKI and often required dialysis [6]. Gul et al reported that, even if perinatal mortality and neonatal morbiditymortality were similar in HELLP syndrome compared with severe preeclampsia-eclampsia without HELLP, overall stillbirth was higher in HELLP syndrome with no regular prenatal care and reported to be as high as $10,3 \%$ [12]. The neonatal mortality rate has been reported to be $19,5 \%$, compared to $2 \%$ in normotensive subjects [13].

Thrombotic Thrombocytopenic Purpura (TTP)

TTP is a rare, life-threatening TMA characterized by severe deficiency in ADAMTS-13 (A Disintegrin And Metalloprotease with ThromboSpondin type 1 domain 13). Pregnancy is a known trigger of TTP; partly due to the physiological decrease of ADAMTS-13 activity, while its substrate, von Willerbrand Factor (vWF), increases [14].

Overall, the estimated incidence of TTP associated with pregnancy is less than 1 per 100,000 pregnancies [15], even if a retrospective study on 8908 deliveries in a tertiary unit reported that the prevalence of TTP among thrombocytopenic pregnant women was up to $5 \%$ [16].

There were 4 pregnancies in this cohort. Regarding perinatal outcome, there were two neonatal deaths due to the prematurity and aorta coarctation. The other two infants were also born prematurely, but the outcome was uneventful.

There are two types of TTP during pregnancy: acquired, antibody mediated TTP and late onset congenital TTP, presenting as de novo in pregnancy. In women with acquired TTP, there is an evidence of anti-ADAMTS 13 IgG antibodies [17], while late onset congenital TTP has been reported to represent up to $66 \%$ of TTP unmasked during pregnancy [18]. It can occur anytime during pregnancy, but the risk is greater in the second and third trimester. Women with intrauterine fetal death or those with previous fetal loss or severe growth restriction have an increased risk [19].

Clinical presentation of congenital and acquired TTP are similar. Typical features of TTP are mainly neurological symptoms, but elevated troponin levels and renal impairment may also be present. Although previous studies described a typical pentad of symptoms suggestive of TTP: fever, hemolytic anemia, thrombocytopenia, renal impairment, and neurologic manifestations, recent studies reported this pentad to be present only in $5-40 \%$ of cases of TTP [20]. Fever seems to be quite uncommon and other reported symptoms are nausea, vomiting, diarrhea, abdominal pain, weakness and bleeding. In general, platelets count is between 20,000 and $50000 / \mathrm{mm}^{3}$ at presentation but it can range between 5000 and $120000 / \mathrm{mm}^{3}$ [1]. The most common complication is the relapse of the condition, reported in up to $36 \%$ of cases [21]. If untreated, the reported mortality may reach $90 \%$ [1].

\section{Atypical Hemolytic Uremic Syndrome (aHUS)}

aHUS is a rare genetic disorder characterized by complementmediated TMA resulting from mutations affecting the regulation of the alternative complement pathway. Typical manifestations are hemolytic anemia, thrombocytopenia and renal impairment.

According to the "multiple-hit" hypothesis [22], aHUS is a consequence of both genetic predisposition to alternative complement dysregulation and occurrence of events or conditions that may precipitate TMA by activating complement and/or damaging the endothelium. Complement-amplifying conditions, such as pregnancy and its complications (preeclampsia, HELLP), may be comorbid with aHUS, unmasking a previously undiagnosed case, or leading to a misdiagnosis [23]. Moreover, aHUS may occasionally 
develop as a complication of preeclampsia, making difficult to differentiate between those conditions.

Pregnancy can unmask aHUS in patients with genetic susceptibility. A French retrospective study reported that in a cohort of 100 adult female aHUS patients, about $20 \%$ of cases were pregnancy-related.

Of all pregnancy-related aHUS, $70-80 \%$ of cases presents in the postpartum period [24]. Indeed, the production of complement regulating proteins by the placenta terminates after delivery [25].

The risk for pregnancy associated aHUS was highest during a second pregnancy in this cohort. [26] A subsequent French study reported that among 87 patients with pregnancy-associated HUS, $76 \%$ developed in the postpartum period and 58\% during the first pregnancy [27].

On contrary, a retrospective study by Huerta et al from the Spanish aHUS Registry reported that majority (16/22) of patients with pregnancy related aHUS presented during the first pregnancy [24]. In the Austrian cohort, out of the 7 patients who presented aHUS during or soon after the pregnancy, 5 (71,4\%) developed aHUS during the first pregnancy [28].

There are several gene mutations related to susceptibility to the disease; these mutations lead to loss of function of several complement regulatory proteins. However, the disease can develop also without any known mutation and the penetrance of complement-aHUS has been found to be only approximately $50 \%$ [29].

There is an emerging evidence that clinical manifestations and prognosis of the aHUS is strongly related to the genotype involved [25]. The influence of each complement abnormality on clinical characteristics and prognosis of aHUS are discussed in details in a review by Yoshida et al. [30] This may be the reason for different outcomes in different cohorts.

While most of the studies in women with aHUS included nonpregnant patients, studies describing pregnancy related aHUS are relatively rare and focused more on the maternal than the neonatal outcome. French cohort reported no differences in outcomes, comparing patients with pregnancy- related and non- pregnancyrelated aHUS [26].

Women carrying genetic mutations are more likely to have preeclampsia compared to the general population (7,7\% vs $2-5 \%)$. However, majority of pregnancies are uneventful [25].
In the Spanish cohort, of six patients that developed aHUS prior to delivery, three delivered near term, one at 32 weeks and one at 24 weeks. The last case had therapeutic abortion due to the fetal hydrops, probably not related to the aHUS. The French cohort reported $4.8 \%$ of fetal loss, but no details were provided. The Vienna Thrombotic Microangiopathy Cohort retrospectively and prospectively investigated 27 maternal and fetal pregnancy outcomes in 14 women with aHUS. Twenty-one pregnancies (78\%) resulted in a live birth, two preterm stillborn and four pregnancies ended up with early spontaneous abortions. Thereafter, nine pregnancies of four women were prospectively followed; six of these pregnancies were treated with prophylactic plasma infusions. Of those, one pregnancy resulted in intrauterine fetal death and seven pregnancies were uneventful [28]. In a patient carrying mutations, the cumulative risk of aHUS is around 18\% [25]. It is important to highlight that a pregnancy-related aHUS can relapse independently to pregnancy.

\section{Differential diagnosis of thrombocytopenia in pregnancy and recognition of TMAs}

Clinical recognition of any TMA requires the documentation of MAHA. These laboratory changes very often occur concomitantly with clinical involvement of at least an organ system. Blood test abnormalities and clinical symptoms TMAs-related are summarized in Fig. 1.

Thrombocytopenia represents the second most common finding after anemia during complete blood count (CBC) in pregnant woman.

Most of thrombocytopenia cases (70-80\%) are gestational thrombocytopenias and deserve no special management, but in presence of an abnormal peripheral blood smear and hemolysis markers further investigation is deemed, as this finding could be associated with a TMA such as HELLP syndrome, TTP or aHUS.

Disseminated Intravascular Coagulation (DIC), usually secondary to infection or malignancy, stands apart from the other TMAs because it has characteristic laboratory abnormalities indicative of a consumptive coagulopathy, including an elevated international normalized ratio (INR) and activated partial thromboplastin time (aPTT).

TMAs due to autoimmune diseases are primarily recognized by the presence of a positive Direct Coombs Test(DCT) [31]. Those TMAs
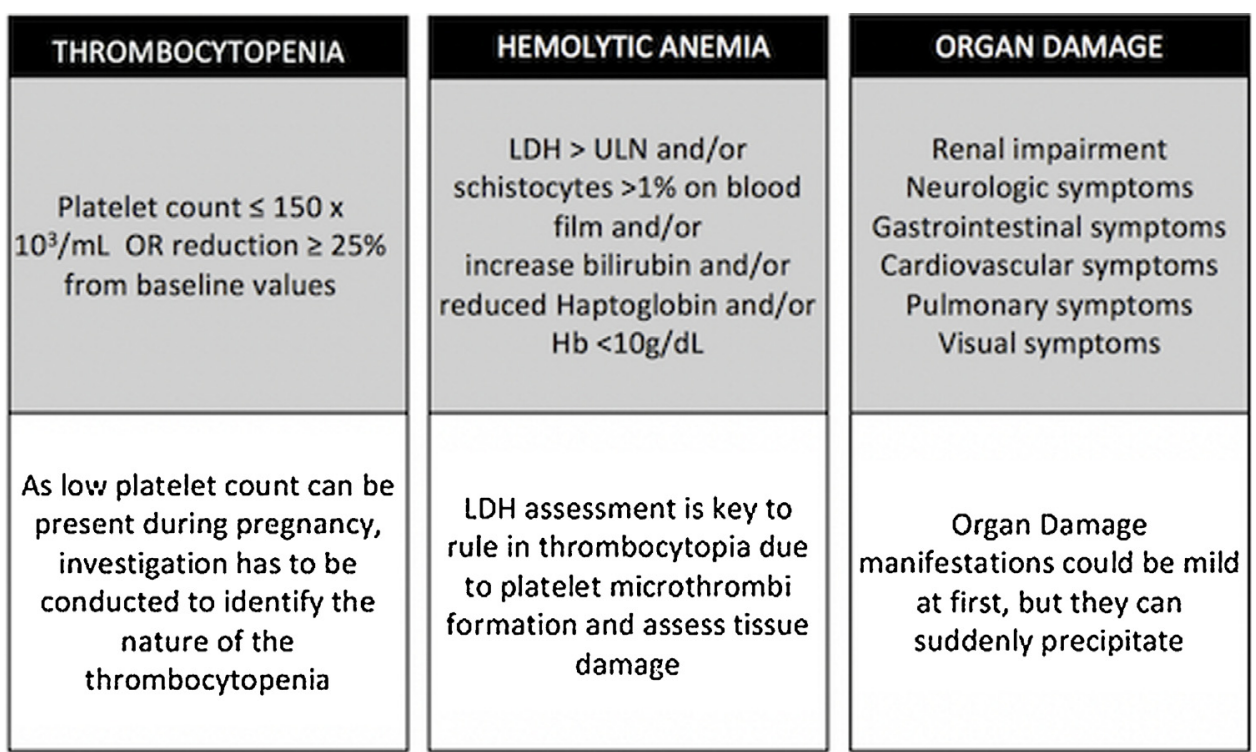

Fig. 1. Laboratory changes and clinical symptoms related to TMAs. ULN (Upper limit of the normal). 
should be managed by addressing the primary disease, such as Systemic Lupus Erythematosus (SLE), Scleroderma or Anti-Neutrophil Cytoplasmic Antibodies (ANCA)-Vasculitis. If those signs and symptoms of TMA do not resolve once the identified condition has been treated, it has to be considered that the primary disease may have unmasked aHUS or TTP and, therefore, undertake appropriate diagnostic procedures to investigate that possibility [23].

\section{HELLP or a different TMA?}

Generally, post-partum full rapid recovery is the rule for HELLP, but therapeutic intervention is needed when hemolysis, thrombocytopenia or renal failure continue to worsen after $48-72 \mathrm{hrs}$ postpartum. It is important to consider TPP or aHUS in the differential diagnosis of HELLP non recovering spontaneously after

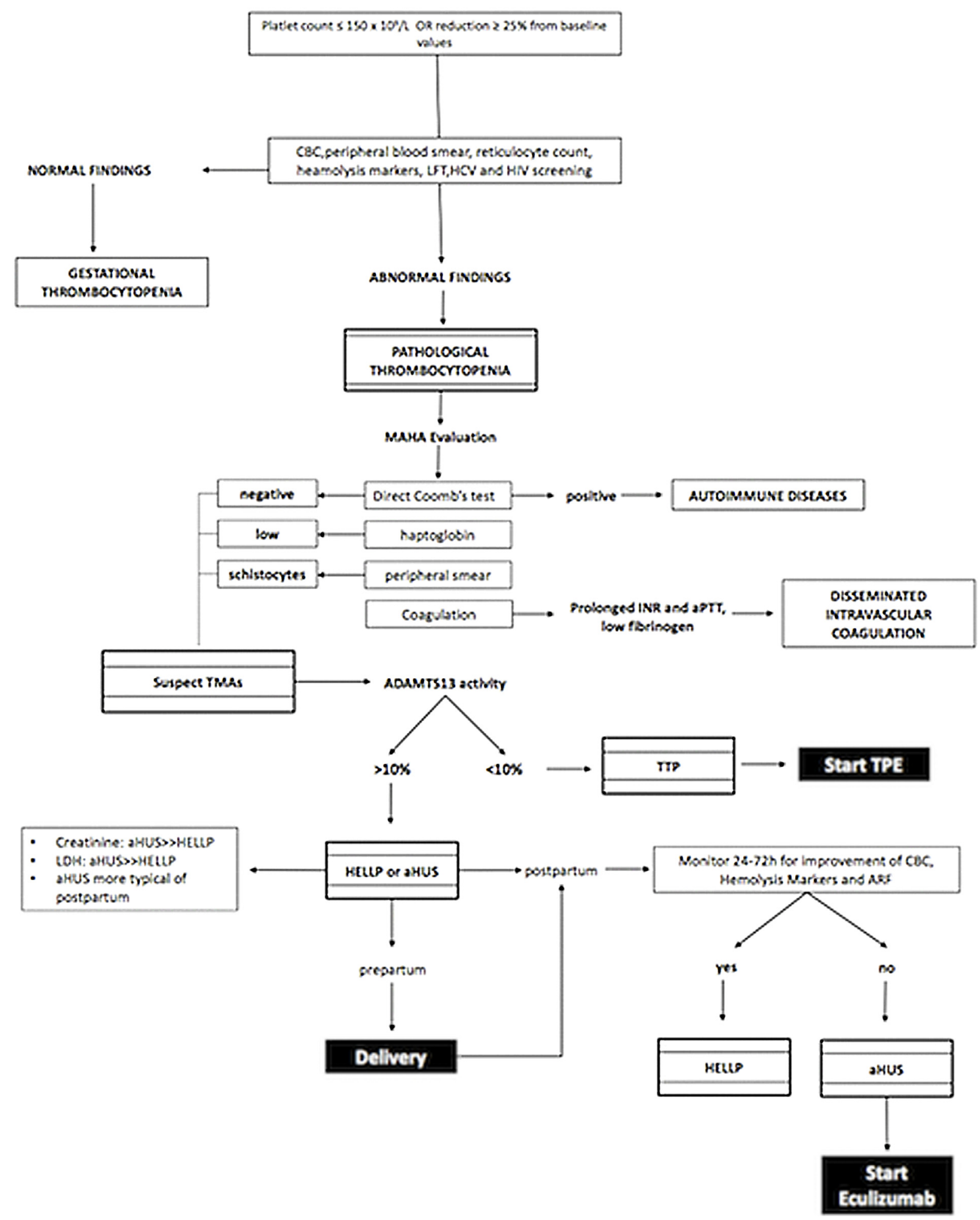

Fig. 2. Simplified flow chart for differential diagnosis of thrombocytopenia in pregnancy and specific management of pregnancy-related TMAs. CBC (cell blood count); LFT (liver function test). 
48-72 h with one or multi-organ involvement, and provide appropriate treatment such as complement inhibitor in case of ADAMTS-13 activity level above 5-10\% and no overt Disseminated Intravascular Coagulation [32].

Moreover, aHUS is mainly characterized by an acute renal failure and hemolysis, with elevated serum creatinine level $(>2 \mathrm{mg} / \mathrm{dl}$ ) and elevated LDH values (>1000 U/l), while these parameters are less abnormal in HELLP syndrome [23].

ADAMTS-13 testing is very relevant in management decision for TMAs, as it has been recently shown that Therapeutic Plasma Exchange (TPE) is not providing any morbidity and/or mortality benefit if ADAMTS-13 is above 10\%, and rapid ADAMTS-13 testing results provide significant cost saving for the hospital due to avoidance of unnecessary aphaeretic procedures for TMA management [33].

\section{Treatment of pregnancy-related TMAs}

It is of essential importance to correctly identify TMAs in pregnancy due to the specific management and treatment for each condition. The American Society for Apheresis (ASFA) provides evidence Category I, Recommendation Grade 1A for Therapeutic Apheresis only for the management of TPP while, in other TMAs, TPE may be ineffective or even harmful and these conditions have been designated Category IV indications for TPE in the current ASFA guidelines [34].

Scully et al [35] have reported five successful pregnancies and deliveries in the third trimester after regular plasma exchange and low molecular weight heparin treatment in patients with history of TTP and very poor neonatal outcome in previous pregnancies. Of note, one patient was treated also with rituximab (a humanized monoclonal antibody against B-cell surface antigen CD20). Subsequently, the same author reported obstetrical and neonatal outcome in a prospective study of TTP cases from the UK TTP registry. Fetal loss occurred in 16/38 pregnancies before cTTP was diagnosed, but in none of the 15 subsequent managed pregnancies [18].

It has to be reminded that TPE is a lifesaving procedure in cases of TPP, but is also invasive, with significant risk of complications, therefore TPE necessitates thoughtful reservation concerning its use as an emergent treatment modality.

Plasmapheresis can be only temporarily or partially effective in the majority of cases of aHUS, with no recovery of renal function in up to $80 \%$ of cases [26].

Eculizumab has been approved for treatment of aHUS and paroxysmal nocturnal haemoglobinuria (PNH). It is a humanized IgG2/4 kappa anti-C5 antibody and acts by blocking the enzymatic cleavage of $\mathrm{C} 5$ to $\mathrm{C} 5 \mathrm{a}$ and $\mathrm{C} 5 \mathrm{~b}$.

It is a major step forward in the treatment of aHUS patients, acting to block complement-mediated inflammation and endothelial damage and leading to a reduction of need for blood transfusions and improving the prognosis [23]. As an example, in the Spanish cohort with pregnancy associated aHUS, seventeen patients underwent plasma treatments with only three having a positive response while ten patients received eculizumab with an excellent renal response in all, independent of carrying or not inherited complement abnormalities [24].

Data on its use during pregnancy are still limited, even if according to reported evidences it seems to be safe for fetus and newborn. Higher doses and/or more frequent eculizumab infusions may be required in some patients to achieve rapid and complete complement inhibition [36].

There is only one case report on eculizumab use in pregnancy with HELLP, although it has not been officially approved for this indication. Prolongation of pregnancy from $26+3$ to $28+6$ (17 days) was achieved [37]. There is a considerable neonatal outcome improvement for each week of gestation in this group of patients, especially when timing of antenatal steroids and magnesium sulphate neuroprotection is optimal. Although the expectant management in pregnancies with HELLP may gain few days and slightly improve the neonatal outcome, it can be associated with severe maternal complications. Therefore, in most of the cases prompt delivery is indicated. It might be possible that in the near future, eculizumab will be a drug of choice for selected pregnant women with the clear signs of complement activation.

Fig. 2 displays flow chart summarizing the clues for differential diagnosis and management in case of thrombocytopenia in pregnancy.

\section{Conclusions}

Pregnancy is a condition that itself amplify complement activity and can unmask TMAs [23,32].

TMAs different from HELLP syndrome should be suspected always when recovery of clinical and serological conditions after delivery does not occur.

Improvements of clinical conditions, resolution of headache, visual symptoms, abdominal pain, increasing platelet counts and resolution of MAHA after delivery allows us to exclude TTP or aHUS. Worsening of thrombocytopenia $72 \mathrm{~h}$ after delivery is suggestive of TTP, while increasing serum creatinine is more specific for aHUS.

Suspicion of TTP or aHUS is fundamental to avoid delay in treatment that can lead to irreversible renal failure or sudden death. Although the obstetricians are usually in the first line of handling these conditions and the awareness of these conditions is prerequisite for the recognition and treatment of TMAs, the multidisciplinary involvement is of essential importance. Eculizumab, as a novel treatment has been approved for aHUS treatment, but it may be feasible also in selected HELLP cases with the clear evidence of the complement activation. Due to the cost of eculizumab, it is unlikely that randomized trials will be soon conducted, but we hope that more case reports will be emerging in the near future.

\section{Acknowledgments}

This research was supported by the The Helsinki University Central Hospital (HUCH) governmental subsidiary (EVO) funds for clinical research (VS)

\section{References}

[1] Kappler S, Ronan-bentle S, Graham A. Thrombotic microangiopathies (TTP, HUS, HELLP). Emerg Med Clin North Am 2014;32(3):649-71.

[2] Mayer SA, Aledort LM. Thrombotic microangiopathy: differential diagnosis, pathophysiology and therapeutic strategies. Mt Sinai J Med 2005;72(May (3)):166-75.

[3] Saito S, Shiozaki A, Nakashima A, Sakai M, Sasaki Y. The role of the immune system in preeclampsia. Mol Aspects Med 2007;28(April (2)):192-209.

[4] Dashe JS, Ramin SM, Cunningham FG. The long-term consequences of thrombotic microangiopathy (thrombotic thrombocytopenic purpura and hemolytic uremic syndrome) in pregnancy. Obstet Gynecol 1998;91(May (5 Pt 1)):662-8.

[5] Wolf JL. Liver disease in pregnancy. Med Clin North Am 1996;80(September (5)):1167-87.

[6] Sibai BM, Ramadan MK, Usta I, Salama M, Mercer BM, Friedman SA. Maternal morbidity and mortality in 442 pregnancies with hemolysis, elevated liver enzymes, and low platelets (HELLP syndrome). Am J Obstet Gynecol 1993;169 (October (4)):1000-6.

[7] Barton JR, Sibai BM. Diagnosis and management of hemolysis, elevated liver enzymes, and low platelets syndrome. Clin Perinatol 2004;31(December (4)):807-33.

[8] Vaught AJ, Gavriilaki E, Hueppchen N, Blakemore K, Yuan X, Seifert SM, et al. Direct evidence of complement activation in HELLP syndrome: a link to atypical hemolytic uremic syndrome. Exp Hematol ISEH - Int Soc Exp Hematol 2016;44(5):390-8.

[9] Salmon JE, Heuser C, Triebwasser M, Liszewski MK, Kavanagh D, Roumenina $\mathrm{L}$, et al. Mutations in complement regulatory proteins predispose to 
preeclampsia: a genetic analysis of the PROMISSE cohort. M. Fisk N, editor. PLoS Med 2011;8(March (3)). . e1001013. Available from: http://dx.plos.org/ 10.1371/journal.pmed.1001013.

[10] Kuklina EV, Ayala C, Callaghan WM. Hypertensive disorders and severe obstetric morbidity in the United States. Obstet Gynecol 2009;113(June (6)):1299-306.

[11] Prakash J, Vohra R, Wani IA, Murthy AS, Srivastva PK, Tripathi K, et al. Decreasing incidence of renal cortical necrosis in patients with acute renal failure in developing countries: a single-centre experience of 22 years from Eastern India. Nephrol Dial Transplant 2007;22(April (4)):1213-7.

[12] Gul A, Cebeci A, Aslan H, Polat I, Ozdemir A, Ceylan Y. Perinatal outcomes in severe preeclampsia-eclampsia with and without HELLP syndrome. Gynecol Obstet Invest 2005;59(2):113-8.

[13] Loirat C, F-B V. Neonatal outcome after preterm delivery in HELLP syndrome. Yonsei Med J 2006;47(3):393-8.

[14] Veyradier A, Stepanian A, ADAMTS Coppo P. Thrombotic thrombocytopenic purpura and pregnancy. Hered Gene. OMICS Int 2013;1(February (S1)):1-7.

[15] Sibai BM. Imitators of severe pre-eclampsia. Semin Perinatol 2009;33(June (3)):196-205.

[16] Delmas Y, Helou S, Chabanier P, Ryman A, Pelluard F, Carles D, et al. Incidence of obstetrical thrombotic thrombocytopenic purpura in a retrospective study within thrombocytopenic pregnant women: a difficult diagnosis and a treatable disease. BMC Pregnancy Childbirth 2015;15(1):1-8.

[17] Tsai H-M, EC-Y Lian. Antibodies to von willebrand factor-cleaving protease in acute thrombotic thrombocytopenic purpura. N Engl J Med 1998;339 (Novemeber (22)):1585-94.

[18] Scully M, Thomas M, Underwood M, Watson H, Langley K, Camilleri RS, et al. Thrombotic thrombocytopenic purpura and pregnancy: presentation, management, and subsequent pregnancy outcomes. Blood 2014;124(July (2)):211-9.

[19] Thomas MR, Robinson S, Scully MA. How we manage thrombotic microangiopathies in pregnancy. Br J Haematol 2016;173(June (6)):821-30.

[20] Ridolfi RL, Bell WR. Thrombotic thrombocytopenic purpura: report of 25 cases and review of the literature. Medicine (Baltimore). Medicine 1981;60 (November (6)):413-28.

[21] Boyce TG, Swerdlow DL, Griffin PM. Escherichia coli 0157:H7 and the hemolytic-uremic syndrome. N Engl J Med 1995;333(August (6)):364-8.

[22] Riedl M, Fakhouri F, Le Quintrec M, Noone DG, Jungraithmayr TC, FremeauxBacchi V, et al. Spectrum of complement-mediated thrombotic microangiopathies: pathogenetic insights identifying novel treatment approaches. Semin Thromb Hemost 2014;40(June (4)):444-64.

[23] Asif A, Nayer A, Haas CS. Atypical hemolytic uremic syndrome in the setting of complement-amplifying conditions: case reports and a review of the evidence for treatment with eculizumab. J Nephrol 2017;30:347-62.
[24] Huerta A, Arjona E, Portoles J, Lopez-Sanchez P, Rabasco C, Espinosa M, et al. A retrospective study of pregnancy-associated atypical hemolytic uremic syndrome. Kidney Int 2017;450-9.

[25] Fakhouri F, Roumenina L, Provot F Sallée M, Caillard S, Couzi L et al. Pregnancy-associated hemolytic uremic syndrome revisited in the era of complement gene mutations. J Am Soc Nephrol 2010;21(May (5)):859-67.

[26] Fakhouri F, Roumenina L, Provot F, Sallee M, Caillard S, Couzi L, et at. Pregnancy-associated hemolytic uremic syndrome revisited in the era of complement gene mutations. J Am Soc Nephrol 2010;21(5):859-67.

[27] Bruel A, Kavanagh D, Noris M, Delmas Y, Wong EKSW, Bresin E, et al. Hemolytic uremic syndrome in pregnancy and postpartum. Clin J Am Soc Nephrol 2017;12(8):1237-47.

[28] Gaggl M, Aigner C, Csuka D, Szilágyi Á, Prohászka Z, Kain R, et al. Maternal and fetal outcomes of pregnancies in women with atypical hemolytic uremic syndrome. . p. 1-10.

[29] Loirat C, Frémeaux-Bacchi V. Atypical hemolytic uremic syndrome. Indian Pediatr 2010;19(5):432-8.

[30] Yoshida Y, Kato H, Ikeda Y, Nangaku M. Pathogenesis of atypical hemolytic uremic syndrome. . p. 1-12.

[31] Barbour T, Johnson S, Cohney S, Hughes P. Thrombotic microangiopathy and associated renal disorders. Nephrol Dial Transplant 2012;27(July (7)):2673-85.

[32] Laurence J. Recognition and treatment of syndromes linked to dysregulation of the alternative complement pathway. Transfus Apher Sci 2016;54(2)179-80 Elsevier Ltd.

[33] Bendapudi PK, Li A, Hamdan A, Uhl L, Kaufman R, Stowell C, et al. Impact of severe ADAMTS13 deficiency on clinical presentation and outcomes in patients with thrombotic microangiopathies: the experience of the Harvard TMA Research Collaborative. Br J Haematol 2015;171(Decemeber (5)):836-44.

[34] Schwartz J, Padmanabhan A, Aqui N, Balogun RA, Connelly-Smith L, Delaney M, et al. Guidelines on the use of therapeutic apheresis in clinical practiceevidence-based approach from the Writing Committee of the American Society for apheresis: the seventh special issue. J Clin Apher 2016;31(June (3)):149-338.

[35] Scully M, Starke R, Lee R, Mackie I, Machin S, Cohen H. Successful management of pregnancy in women with a history of thrombotic thrombocytopaenic purpura. Blood Coagul Fibrinolysis 2006;17(6):459-63.

[36] Sarno L, Tufano A, Maruotti GM, Martinelli P, Balletta MM, Russo D. Eculizumab in pregnancy: a narrative overview. J Nephrol 2018 Springer International Publishing.

[37] Burwick RM, Feinberg BB. Eculizumab for the treatment of preeclampsia/ HELLP syndrome. Placenta 2013;34(2)201-3 Elsevier Ltd. 